\title{
BRIGHTEST STARS IN GALAXIES AS DISTANCE INDICATORS
}

\author{
A1lan Sandage \\ Carnegie Institution of Washington, Department of Astronomy \\ 813 Santa Barbara St., Pasadena, CA 91101-1292
}

\section{ABSTRACT}

The current status of the absolute magnitude calibration of the brightest blue and red supergiants in galaxies of different absolute magnitudes shows trends of $\mathrm{M}$ (star) with M(parent galaxy). Red supergiants show a more shallow correlation than the blue stars for galaxies brighter than $\mathrm{M}_{\mathrm{B}}=-14$. For fainter galaxies, the red supergiant method appears to become totally degenerate.

Four areas of application of the brightest star data are discussed as (1) determining $\mathrm{M}_{B}$ of the Eddington limit to be $\sim-10$ for blue supergiants, (2) calibration of $\mathrm{M}_{B}(\max )=-20.0 \pm 0.4$ for type I supernovae, leading to a Hubble constant of $\mathrm{H}_{\mathrm{O}}=43 \pm 10 \mathrm{~km} \mathrm{~s}^{-1} \mathrm{Mpc}^{-1}$, (3) detection of the deceleration of the cosmological expansion by the Local Group leading to a Local Group mass of $4 \times 10^{11} \mathrm{MO}$ and a mass-to-blue light ratio of 3, and (4) use of the brightest stars to map the Virgo cluster velocity perturbation of the Hubble flow.

\section{INTRODUCTION}

Persistently, one of the most difficult problems in astronomy has been the determination of accurate distances to external galaxies. The only two fundamental methods we know depend either on (1) properties of the stellar content such as luminosities of variables, brightest stars, normal novae, supernovae, linear sizes of clusters, associations, H II regions, etc., or (2) properties of the dynamics via Opik's (1922) method as applied in its restricted form by Tully and Fisher.

For distances beyond the Local Group and outward into the general field where the cosmological redshift dominates over local streaming motions, Hubble depended only on brightest "stars." His first calibration (Hubble 1926) of $\mathrm{M}_{\mathrm{B}}$ (stars) was essentially his last (Hubble 1936) at $\mathrm{M}_{\mathrm{B}}$ (brightest) $=-6.1$ based on the brightest resolved stars in only a few of the nearest galaxies (M31, M33, LMC, SMC, and NGC 6822--and 
strangely M101 for incorrect reasons) whose distances he already had determined from Cepheids.

In 1950, as Baade was revising Hubble's M31 distance outward by a factor of $\sim 2$, Hubble remarked how ironic it had turned out that from 1924 to the late-1940's his distance scale was thought to be much too large by stellar spectroscopists everywhere; no one had believed that they had ever seen stars brighter than Mpg $\simeq-3$ spectroscopically. The calibration of distances in our own galaxy has also undergone a drastic revision over the past 30 years, just as has the extragalactic distance scale. The brightest Galactic stars are now indeed put near $M_{B} \simeq-10$, which is the current value from the external galaxies as well.

Hubble's work that ended in 1936 gave an expansion constant of $\mathrm{H}_{\mathrm{O}}=530 \mathrm{~km} \mathrm{~s}^{-1} \mathrm{Mpc}^{-1}$ which is 10 times ( 5 magnitudes) larger than the current value. His problems were: stars,

a) use of $M_{B}=-6$ rather than -10 for the brightest blue

b) incorrect magnitude scales in the Selected areas such that stars 1isted as $\mathrm{m}_{\mathrm{pg}} \simeq 18-20$ are actually at $\mathrm{B} \simeq 20-22$ (Stebbins, Whitford, and Johnson 1950; Sandage 1983a, Fig. 5, 1983b, Fig. 6), c) real stars in his galaxies start near $B \simeq 22$ (in Virgo) rather than his 19.5, due partly to item (b) and partly because the objects he identified as brightest stars in remote galaxies are not stars but are rather compact H II regions or small associations.

\section{CALIBRATION OF THE BRIGHTEST STARS}

Beginning in 1950 when the Hale $5 \mathrm{~m}$ reflector was put into routine use, a long-range program of faint photometry was begun in nearby galaxies as the next step. The aim has been to establish an accurate distance scale to calibrate the cosmological expansion rate. The work has proceeded along two separate routes. (1) Relative distances of clusters of galaxies over a redshift range of $0.003<z \leqslant 0.4$ were established by measuring total magnitudes of the brightest $\mathrm{E}$ galaxies in them.

(2) Measurement of absolute distances to highly resolved nearby galaxies was attempted via Cepheids and brightest stars so as to walk along the way to the Virgo cluster.

The Cepheid P-L relation was recalibrated using Cepheids in galactic clusters (Sandage and Tammann 1968) and then applied to NGC 2403 directly (Tammann and Sandage 1968) and M81 and M101 indirect1y. In the summary of the brightest star calibration as it existed from this early work (Sandage and Tammann 1974), the brightest red supergiants were isolated as a new distance indicator. From the data available at that time we concluded that $\mathrm{M}_{\mathrm{V}}(1)=-7.9 \pm 0.1$ mag for these red stars, independent of the parent galaxy. We had only 8 calibrators (the Galaxy, LMC, SMC, NGC 6822, IC 1613, NGC 2403, IC 2574, and Ho II) of which only 5 were based on Cepheids. 
In studying the blue supergiants we also produced a calibration based on 12 galaxies in which $\left\langle M_{B}(1)>\right.$ varies from -8 to -9.5 as the parent galaxy changed from Mpg $=-14.4$ to -19 , an effect first discussed extensively by Holmberg (1950).

The work since 1974 has been aimed at improving these values by increasing the sample with Cepheids, and also by strengthening the internal photometry. Cepheids have been found and worked up in the faint Im dwarfs of Sextans A and Sextans B, and WLM (Sandage and Carlson 1985a,b). Holmberg IX, the companion to M81 at a common distance of $\mathrm{m}-\mathrm{M}=28.8$, has a color-magnitude diagram (Sandage 1984). Cepheids have been found in the Pegasus dwarf, in Leo A, NGC 3109, and IC 5152 and are currently under study in the latter two. Cepheids in NGC 300 have been studied by Graham (1984), and photometry of the brightest red and blue stars is nearly completed (see also Graham and Humphreys, this volume for their independent value of $\left\langle\mathrm{M}_{\mathrm{v}}(4)\right\rangle=-7.9$ for four red supergiants).

TABLE 1

Summary of the Absolute Magnitudes of the Brightest Red and Blue Stars

\begin{tabular}{|c|c|c|c|c|c|c|c|}
\hline $\begin{array}{l}\text { Name } \\
(1)\end{array}$ & $\begin{array}{c}\mathrm{M}_{\mathrm{B}} \text { (parent) } \\
(2)\end{array}$ & $\begin{array}{l}B(3) \\
(3) \\
\end{array}$ & $\begin{array}{c}(\mathrm{m}-\mathrm{M}) \mathrm{AB} \\
(4)\end{array}$ & $\begin{array}{c}M_{B}(3) \\
(5) \\
\end{array}$ & $\begin{array}{l}\mathrm{V}(3) \\
(6) \\
\end{array}$ & $\begin{array}{c}(m-M) A V \\
(7)\end{array}$ & $\begin{array}{r}\mathrm{M}_{\mathrm{V}}(3) \\
(8) \\
\end{array}$ \\
\hline 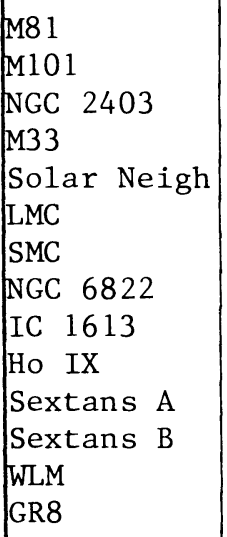 & $\begin{array}{l}-22.8 \\
-21.3 \\
-19.5 \\
-19.0 \\
-18.5 \\
-18.4 \\
-16.8 \\
-15.8 \\
-14.7 \\
-14.5 \\
-14.3 \\
-14.3 \\
-13.5 \\
-11.3\end{array}$ & $\begin{array}{r}19.1 \\
19.3 \\
18.1 \\
15.4 \\
\cdots . . \\
9.5 \\
10.6 \\
16.9 \\
16.7 \\
20.1 \\
18.7 \\
19.4 \\
17.7 \\
(18.4)\end{array}$ & $\begin{array}{l}28.8 \\
29.2 \\
27.9 \\
25.3 \\
\cdots \cdots .9 \\
18.9 \\
19.3 \\
25.0 \\
24.6 \\
28.8 \\
26.2 \\
26.2 \\
24.9 \\
25.2\end{array}$ & $\begin{array}{l}-9.7 \\
-9.9 \\
-9.8 \\
-9.9 \\
-8.8 \\
-9.4 \\
-8.7 \\
-8.1 \\
-7.9 \\
-8.7 \\
-7.5 \\
-6.8 \\
-7.2 \\
(-6.8)\end{array}$ & $\begin{array}{l}19.7 \\
20.5 \\
27.8 \\
16.6 \\
\cdots . . \\
11.1 \\
11.8 \\
16.9 \\
17.0 \\
20.6 \\
18.3 \\
18.9 \\
17.9 \\
(21.0)\end{array}$ & $\begin{array}{l}28.7 \\
29.2 \\
19.4 \\
25.0 \\
\cdots . . \\
18.8 \\
19.3 \\
24.8 \\
24.5 \\
28.7 \\
26.2 \\
26.2 \\
24.9 \\
25.2\end{array}$ & $\begin{array}{l}-9.0 \\
-8.7 \\
-8.4 \\
-8.4 \\
-8.0 \\
-7.7 \\
-7.5 \\
-7.9 \\
-7.5 \\
-8.1 \\
-7.9 \\
-7.3 \\
-7.0 \\
(-4.2)\end{array}$ \\
\hline
\end{tabular}

The calibration of the brightest red and blue stars now available is set out in Table 1 and plotted in Figures 1 and 2, taken from the summary plots given elsewhere from the last paper of the series (Sandage and Carlson 1985b). The principle features of the diagrams are (1) the luminosity of the blue stars progressively decreases with M(parent) when the parent galaxies are fainter than $\mathrm{M}_{\mathrm{B}} \simeq-19$. (2) In the brightest four galaxies in the sample (M81, M101, NGC 2403, M33) the brightest stars 


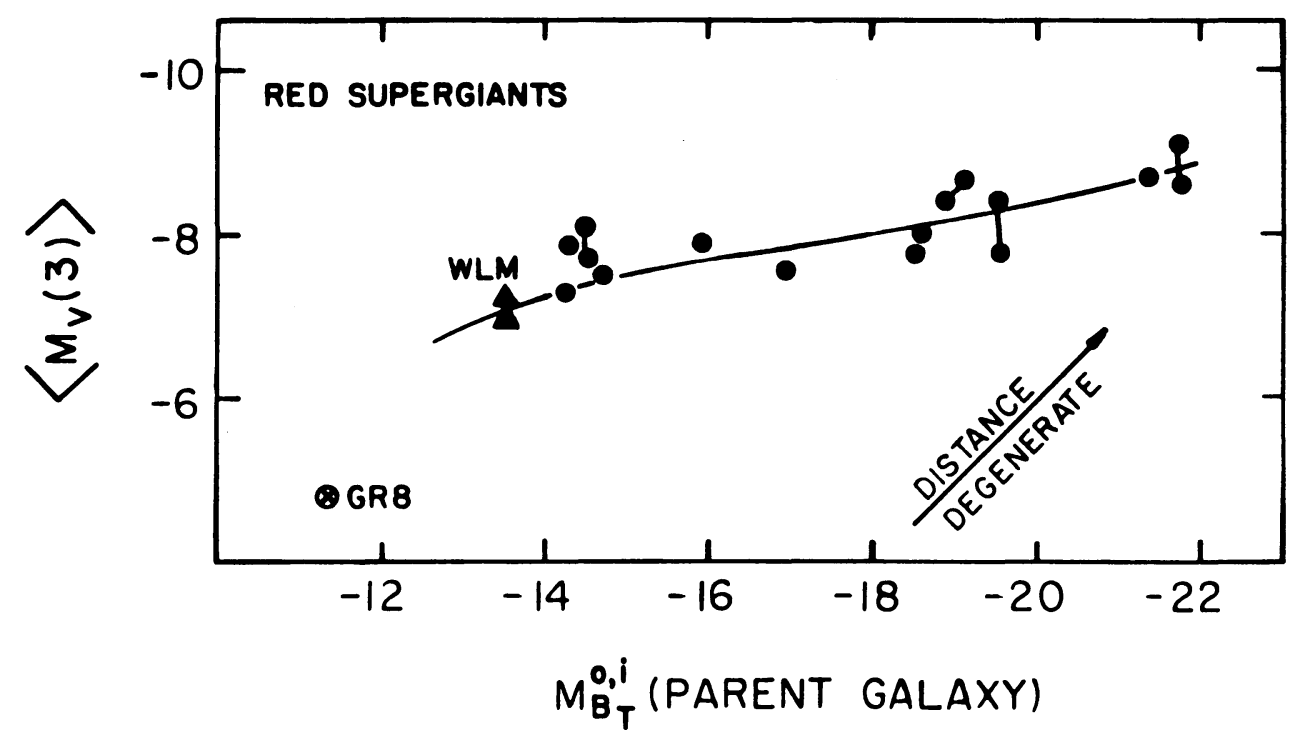

Figure 1. Calibration of the mean absolute visual magnitude of the three brightest red supergiants in the calibrating galaxies in Table 1 .

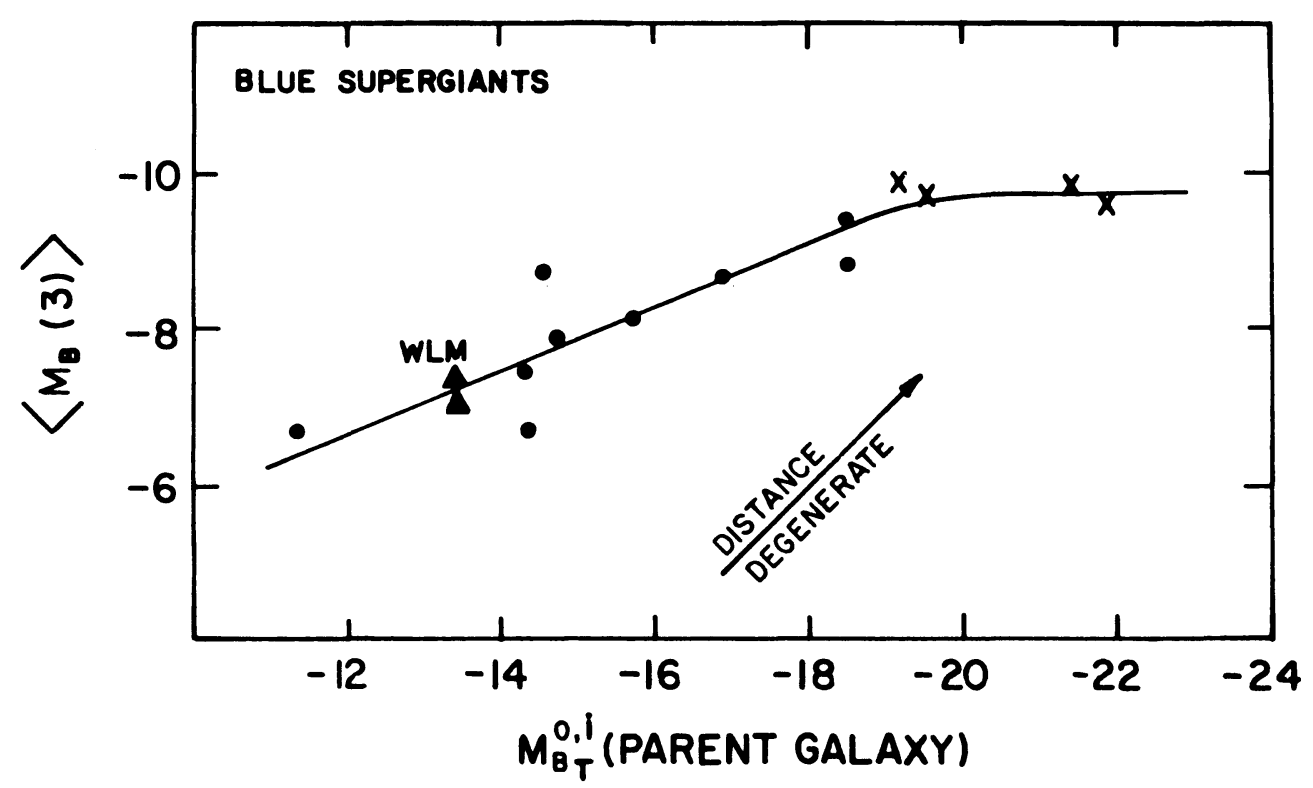

Figure 2. Same as Figure 1 for the $B$ absolute magnitude of the three brightest blue supergiants. 
are irregular blue variables that have characteristic amplitudes of $\Delta \mathrm{B} \simeq$ $1 \mathrm{mag}$ and characteristic time scales of variability of $\sim 10$ to 30 years (Hubble and Sandage 1953). (3) The rate of change of $\mathrm{M}_{B}$ (star) with $\mathrm{M}_{B}$ (galaxy) is less steep than the distance-degenerate line along which there would be no distance information. It is the intersection of the line of slope 1 with the intrinsic calibration line from which the distance to an unknown galaxy can be determined. (4) However, the scatter of the points for the 13 calibrating galaxies about the mean line is large. Such a scatter is expected for the fainter galaxies due to stochastic sampling problems of the luminosity function (Greggio this volume and 1986), amounting to at least $\pm 0.7 \mathrm{mag}$. This stochastic problem makes use of the brightest blue stars as distance indicators less attractive than the red supergiants shown in Figure 2. (5) The red supergiant calibration shows less dependence of $\left\langle\mathrm{M}_{\mathrm{V}}(3)\right\rangle$ on $\mathrm{M}_{\mathrm{B}}$ (parent) than for the blue stars. Nevertheless, there is a dependence with a mean slope of $\mathrm{dM}_{\mathrm{V}}($ star $) / \mathrm{dM}_{\mathrm{B}}$ (parent) $\simeq 0.2$. (6) If the data for GR8 are correct, the red star relation becomes entirely degenerate for $M_{B}$ (parent) fainter than $\simeq-14$ because the intrinsic line that connects WLM with GR8--not shown in Figure 2 has the degenerate slope of 1 . This is the problem we faced in Leo A and the Pegasus dwarf (Sandage 1986), showing that for dwarf galaxies fainter than $\mathrm{M}_{\mathrm{B}} \simeq-14$ the brightest red supergiants may not be distance indicators at a11.

\section{APPLICATION OF THE BRIGHTEST STAR DATA}

Four areas of application of the data on the brightest stars and the distances to their parent galaxies can be mentioned.

3a. Determining $\mathrm{M}_{\mathrm{B}}$ for the Eddington Limit

If the data for M81, M101, NGC 2403, and M33 are representative, the brightest blue stars may define the upper limit of stability for stars as they have just moved off the main sequence. The two suggestive reasons are: (1) the stars are variable as mentioned before, and (2) there is no correlation of $M_{B}$ (star) with $M_{B}$ (parent galaxy) for them as there is for fainter galaxies (Figure 2). The explanation of the slope in Figure 2 for $\mathrm{M}_{\mathrm{B}}$ (parent galaxy) fainter than -19 is undoubtedly the purely statistical effect of sampling the stellar luminosity function at fainter magnitudes with the condition that $\phi(M)=1$, as the normalization constant for $\phi(M)$ becomes smaller for fainter parent galaxies (Holmberg 1950; Sandage and Tammann 1974). The leveling off of the $\mathrm{M}_{\mathrm{B}}($ star $)=\mathrm{f}[\mathrm{M}(\mathrm{galaxy})]$ correlation brighter than $\mathrm{M}_{\mathrm{B}}$ (galaxy) $=-19$ then requires $\phi(M)$ star to have a vertical cut off at $M_{B} \cong-10$. The most reasonable explanation is an instability, which is the Eddington limit where the radiation pressure equals the gas pressure. As these variables are of spectral type $\mathrm{F}$ with $\mathrm{T} \simeq 10,000^{\circ} \mathrm{K}$, the bolometric correction is sma11, suggesting $\mathrm{M}_{\mathrm{bol}} \simeq-10.5$ as this 1 imit. 
3b. Calibration of $\mathrm{M}_{\mathrm{B}}(\max )$ for $\mathrm{SNe} I$ as a Distance Indicator

Evidence is growing that most supernovae of type I are standard explosions with a tightly fixed absolute luminosity at maximum. The main points (Cadonau, Sandage, and Tammann 1985) are (1) the Hubble diagram is well defined with a scatter in magnitude about the linear velocity-distance relation that can be due entirely to observational error (Kowal 1968; Sandage and Tammann 1981, Fig. 20), (2) near identity of the light curve shapes at any given time after maximum (Tammann 1978; Elias et al. 1981), and (3) near identity of the spectra at any given phase (cf. Wheeler 1985). Although there may be a small subset ( $10 \%)$ of anomalous type I SNe (Panagia 1985), these can be identified and removed from the sample.

To determine the Hubble constant from the observed redshift-apparent magnitude relation requires calibration of $\mathrm{M}_{B}(\mathrm{SN})$ at maximum. The purely astronomical method is to determine distances to galaxies which have had well observed SNe I. This has been done (Sandage and Tammann 1982) for two galaxies (NGC 4214 and IC 4182) using the brightest resolved stars and the calibrations of Figures 1 and 2 here, and revised for internal absorption in the two galaxies (Cadonau et al. 1985) to give $\mathrm{M}_{\mathrm{B}}(\max )=-20.1 \pm 0.4$. This is in good agreement with the value of $-19.8 \pm 0.7$ from the expansion parallaxes (Arnett 1982; Branch 1982) and with theoretical calculations from an increasingly realistic model of the explosion (Sutherland and Wheeler 1984), giving $M_{B}=-20.0 \pm 0.2$. If $\mathrm{M}_{B}(\max )=-20.0 \pm 0.4$, the magnitude-redshift relation requires the Hubble constant to be $\mathrm{H}_{\mathrm{O}}=43 \pm 10 \mathrm{~km} \mathrm{~s}^{-1} \mathrm{Mpc}^{-1}$.

3c. Deceleration of the Cosmological Expansion by the Loca1 Group

The mass of the Local Group must retard the cosmological expansion, causing the effective Hubble constant to increase outward over distances where the effect is non-negligible. Calculations (Sandage 1986) show that the deceleration caused by the Local Group should be measurable within a distance of $\sim 5 \mathrm{Mpc}$ provided that the distances to the test ga1axies can be determined with high accuracy. Using the galaxies that have gone into the calibration of the brightest stars in Table 1 gives a suggestive result that the deceleration has been found. The best-fit mass of the Local Group obtained by comparing the family of decelerating. velocity-distance relations with the observations is $4 \times 10^{11} \mathrm{MO}$, with a firm upper limit of less than $3 \times 10^{12} \mathrm{MO}$. This gives a best-fit mass-tolight ratio of 3 (in solar blue units) and an upper limit of $\mathrm{M} / \mathrm{L}<20$.

\section{3d. Use of Brightest Stars to Map the Virgo Perturbation}

Precise distances to galaxies strategically placed in angle and distance from Virgo are needed to map the perturbation of the cosmological expansion field caused by the overdensity of the Virgo cluster 
complex. Brightest stars are expected to be an important distance indicator for galaxies beyond the reach of the Cepheids. Lists and three atlases of galaxies closer than $\sim 50 \mathrm{Mpc}$ have been prepared for use in planning observing programs with Space Telescope (Sandage 1985a,b,c).

To map the velocity perturbation it is required to determine the distances to many galaxies by a fundamental (nonredshift) method, and then to compare the observed redshifts with those predicted from a nonperturbed cosmological velocity field. Particular directions and distances from Virgo are advantageous to maximize the perturbation signal. Figure 3 illustrates this problem using a version of the Tonry-Davis (1981) diagram calculated for an "infal1" velocity toward Virgo of 220 $\mathrm{km} \mathrm{s}^{-1}$. Dotted lines are the unperturbed Hubble flow velocities; solid lines are the contours of the perturbed velocity due to Virgo. Shown as crosses are galaxies from the first atlas where resolution into stars is expected to be easy, even from the ground in some cases.

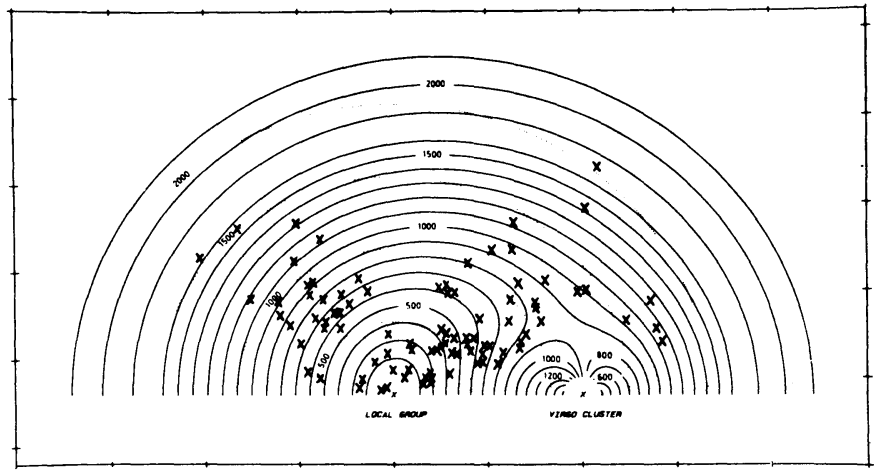

Figure 3. A Tonry-Davis diagram for a Virgo "infall" velocity of 220 $\mathrm{km} \mathrm{s}^{-1}$. Crosses are ga1axies from the first atlas of candidates for resolution into brightest stars.

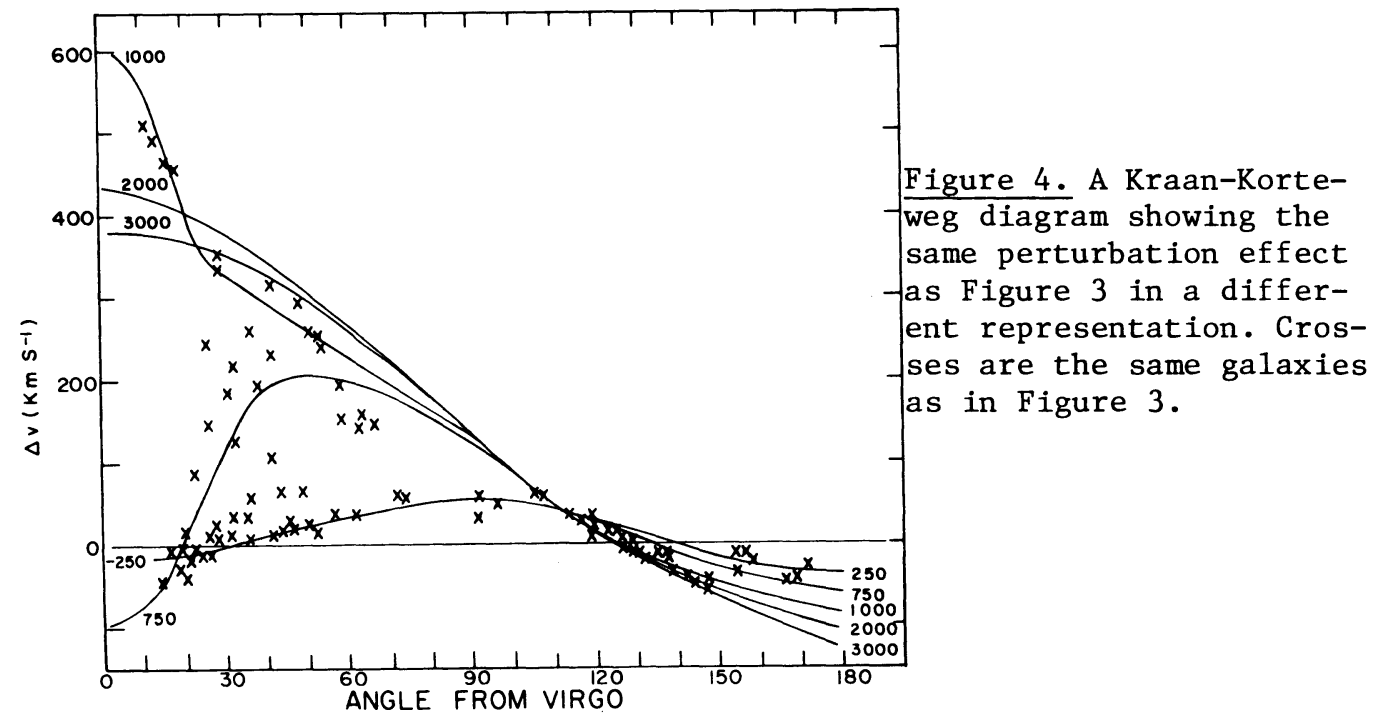


Figure 4 illustrates the same effect in a different representation due to Kraan-Korteweg (1986). Shown as ordinate is the velocity difference between the ideal Hubble flow and the perturbed field as a function of angle from Virgo and the observed velocity. The same galaxies as in Figure 3 are plotted.

More distant galaxies which are more difficult to resolve but whose brightest stars can be studied with Space Telescope are shown in Figure 5 .

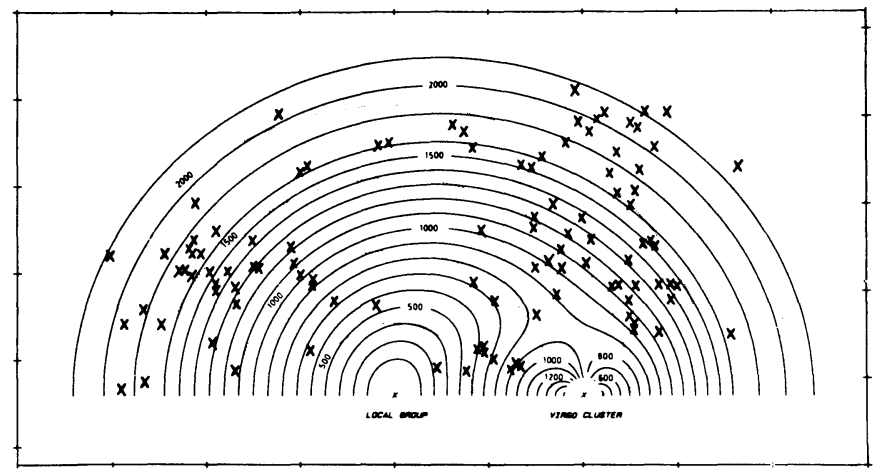

Figure 5. Same as Figure 3 but showing galaxies from the second atlas of candidates. These galaxies are more distant those in Figure 3 .

Finally, Figure 6 is a cautionary note showing the very small signal which must be detected in order to see the perturbation field. What is measured by any method of distance determination is the percentage signal, $\Delta \mathrm{r} / \mathrm{r}=\Delta \mathrm{v} / \mathrm{v}$. This, calculated from Figure 4, is plotted in Figure 6 as a function of angle from Virgo and of the measured velocity.

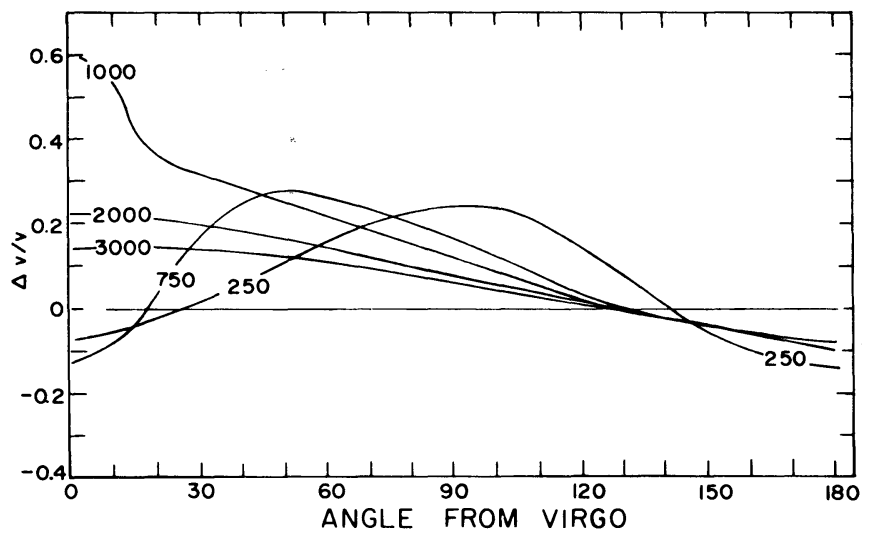

Figure 6. The percentage signal $\Delta v / v$ that must be detected to measure the perturbation, calculated from Figure 4. The photometric difference to be detected is $\Delta$ mag $=$ $2.17 \Delta \mathrm{v} / \mathrm{v}$. 
This signal $\Delta v / v$ translates into a photometric difference of $\Delta$ mag $=2.17$ $\Delta v / v$, showing that we must detect differences of at most only 0.5 mag in order to map the perturbation using photometric methods. The scatter in Figures 1 and 2 shows that the job using brightest stars will be difficult.

\section{REFERENCES}

Arnett, W.D.: 1982, in Supernovae: A Survey of Current Research, eds. M. J. Rees and R.J. Stoneham (Doredrecht: Reide1), p. 221 .

Branch, D.: 1982, in Supernovae: A Survey of Current Research, eds. M.J. Rees and R.J. Stoneham (Dordrecht: Reide1), p. 267.

Cadonau, R., Sandage, A., and Tammann, G.A.: 1985, in Lecture Notes in Physics, No. 224, ed. N. Barte1 (Berlin: Springer), p. 151.

Elias, J.H., Froge1, J.A., Hackwe11, J.A., and Persson, S.E.: 1981, Ap. J. Letters, 251, L13.

Graham, J.A.: 1984, A.J., 89, 1332.

Greggio, L.: 1986, Astron. Astrophys., in press.

Holmberg, E.: 1950, Medd. Lunds Obs. Ser. 2, No. 128.

Hubble, E.: 1926, Ap. J., 64, 321.

Hubble, E.: 1936, Ap. J., $\overline{\overline{84}}, 270$.

Hubble, E., and Sandage, A.: 1953, Ap. J., 118, 353.

Kowa1, C.T.: 1968, A.J., 73, 1021.

Kraan-Korteweg, R.: 1986, Astron. Astrophys., in press.

Öpik, E.: 1922, Ap. J., 55, 406.

Panagia, N.: 1985, in Lecture Notes in Physics No. 224, ed. N. Barte1 (Berlin: Springer), p. 14 .

Sandage, A.: 1983a, in IAU Co11. No. 100, Kinematics, Dynamics, and Structure of the Milky Way, ed. W.L.H. Schuter (Dordrecht: Reide1), p. 315 .

Sandage, A.: $1983 \mathrm{~b}$, A.J., $88,1108$.

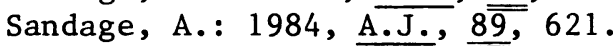

Sandage, A.: 1985a,b,c, A.J., 90, in press (October issue).

Sandage, A.: 1986, Ap. J., in press.

Sandage, A. and Car1son, G.: 1985a, A.J., 90, 1019.

Sandage, A. and Carlson, G.: 1985b, A.J., $\overline{\overline{90}}, 1464$.

Sandage, A. and Tammann, G.A.: 1968, Ap. J., 151, 531.

Sandage, A. and Tammann, G.A.: 1974, Ap. J., $\overline{191}, 603$.

Sandage, A. and Tammann, G.A.: 1981, in Astrophysical Cosmology, Proc.

of the Study Week on Cosmology and Fundamental Physics, ed. H.A.

Brück, G.V. Coyne, and M.S. Longair (Rome: Specola Vaticana), p. 23 .

Sandage, A. and Tammann, G.A.: 1982, Ap. J., 256, 339.

Stebbins, J., Whitford, A.E., and Johnson, H. Sutherland, P.G. and Wheeler, J.C.: 1984, Ap. J., 280, 282.

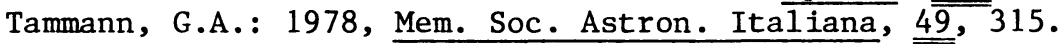

Tammann, G.A, and Sandage, A.: 1968, Ap. J., 151, 825.

Tonry, J.L. and Davis, M.: 1981, Ap. J., 246, 680 .

Wheeler, J.C.: 1985, in Lecture Notes in Physics, No. 224, ed. N.

Barte1 (Berlin: Springer), p. 34. 
Discussion : SAINDAGE.

\section{HUMPHREYS :}

It is important to understand where the difference in the luminosity calibration of the $H$ supergiants comes from. We use different methods to derive the luminosities. Sandage uses the apparent modulus with the apparent magnitude of the brightest red supergiants to derive their $\mathrm{H}_{\mathrm{y}} \mathrm{s}$. I use the true distance modulus corrected for reddening with the visual magnitudes of the stars corrected for the individual reddening affecting each star. If the reddening were uniform then one might expect the two methods to give very similar results; however in M33 the reddening is both significant and variable.

Comparison of the " $\mathrm{V}$ 's with the $\mathrm{M}_{\mathrm{K}}$ and $1 \mathrm{I}_{\mathrm{Bol}}$ measured from infrared photometry shows that when a reddening correction is not applied the resulting $(\mathrm{V}-\mathrm{K})$ and $\mathrm{BC}_{\mathrm{V}}$ (the bolometric correction at $\mathrm{V}$ ) are too blue and too small respectively. $(\mathrm{V}-\mathrm{K})_{\mathrm{Q}}$ and $\mathrm{BC}_{\mathrm{V}}$ derived from Sandage's method for $\mathrm{M}_{\mathrm{V}}$ are not physically meaningful for $M$ supergiants.

\section{SANDAGE :}

There is much less difference than it seems at first between Humphrey's reply here and the calibration of $\left\langle M_{V}(3)\right\rangle$ vs $M$ (parent galaxy) for the red supergiants given in the main report. My main point is that $\left\langle i \mathrm{v}_{\mathrm{v}}(3)\right\rangle$ is not constant at $+7.9 \pm 0.1$ as we originally thought and as Humphreys has now adopted. Räther, $\left\langle H_{\mathrm{Y}}(3)\right\rangle$ for the red supergiants becomes fainter as 11 (parent galaxy) becomes fainter over the entire range of $M_{3}$ (parent) between - 22 and -13.5 . Humphreys obtains the same relation even with her individual adopted correction for $A_{Y}$. If she were to plot her 1101 point in the $\left\langle M_{V}(3)\right\rangle$ vs $M_{B}$ (parent) correlation at her value of $\left\langle i_{\mathrm{V}}(3)\right\rangle=-9.0$ and if she were to decide to use the M81 apparent modulus of $(m-M)_{A B}=28.8$, giving $\left\langle M_{V}(3)\right\rangle=-8.8$, our respective diagrams would be nearly the same. There is, then, no argument except over the value of $\mathrm{m}-\mathrm{M}=28.8$ for $\mathrm{M} 81$.

There is no question that $(m-M))_{3}=27.55$ used by Humphreys is too small by approximately 1 mag. The Cepheids and novae show that the apparent inodulus of 131 is considerably fainter than that for NGC 2403. One will of course obtain an incorrect value of $\left\langle M_{B}(3)\right\rangle$ for the blue stars, and $\left\langle M_{Y}(3)\right\rangle$ for the red stars, by using the same modulus for both 1481 and NGC2403.

This then brings us to the question of the absorption. If the absorption is the same in the mean between the Cepheids (from which the apparent modulus is derived) and the red supergiants, the correct $M_{Y}$ (RSG) is always obtained by combining the apparent modulus (m-M) $\mathrm{AV}$ with the apparent magnitude of the RSG no matter how high Av might be. An error is introduced only if $\left\langle A_{V}\right.$ (Cepheids) $\rangle$ is different from $\langle A \mathrm{~A}(\mathrm{RSG})\rangle$, i.e. in the presence of differential absorption. In M33 there is no evidence for large differential absorption between Hubble's 
Cepheids near the center and our new Cepheids in Field 25. Hence the question of absorption (the main point of disagreement between Humphreys and myself) is not the issue.

Fainter galaxies, such as IC 1403, Sextans A, Sextans B, WLA, (etc) where the Av values themselves are expected tu be very small, clearly show $\left\langle H_{Y}(3)\right\rangle$ for the RSG to be fainter than -7.9 . In WLM at $M_{B}$ (parent) $=-13.5,\left\langle H_{V}(3)\right\rangle=-6.7$. Hence, there is a faintward trend of the RSG in fainter galaxies.

In summary, absorption is not the cause of the apparent difference between Humphreys and Sandage. Rather it is caused by (1) Humphreys' use of the small modulus of 27.55 for M81 for which there is no observational justification, (2) her neglect of the Ml01 point at $\left\langle M_{V}(3)\right\rangle=-9.0, M_{B}$ (parent) $=-22$, and (3) her neglect of the data for WLM, Sextans $A$ and Sextans $B$ which give $\langle M(3)\rangle$ near -7.0 rather than 7.9.

Finally one should note that if 1.181 were put at $(\mathrm{m}-\mathrm{M})_{\mathrm{AB}}=-27.5$ then the 3 brightest blue irregular variables in $M 31$ would each be approximately 1.3 magnitude fainter than -9.9 and they would not fit the blue supergiant calibration where now in M101, M81, M33 and NGC2403 $\left\langle M_{B}(3)\right\rangle=-9.9 \pm 0.1$ using the distances listed in the contribution. One would then have to say that the brightest blue irregular variables in any given galaxy are not at, or very near the de Jager-HumphreysDavidson upper limiting line in the HRD. This, of course, would be unfortunate. But that conclusion is not necessary if $(\mathrm{m}-\mathrm{M}) \mathrm{AB}=28.8$ for M81 as the Cepheid and novae data require. 